\title{
MATRIX RINGS AND ITS TOTAL RINGS OF FRACTIONS
}

\author{
Sang Cheol Lee
}

Abstract. Let $R$ be a commutative ring with identity. Then we prove

$$
\begin{aligned}
M_{n}(R)= & G L_{n}(R) \\
& \cup\left\{A \in M_{n}(R) \mid \operatorname{det} A \neq 0 \text { and } \operatorname{det} A \notin U(R)\right\} \\
& \cup Z\left(M_{n}(R)\right)
\end{aligned}
$$

where $U(R)$ denotes the set of all units of $R$. In particular, it will be proved that the full matrix ring $M_{n}(F)$ over a field $F$ is the disjoint union of the general linear group $G L_{n}(F)$ of degree $n$ over the field $F$ and the set $Z\left(M_{n}(F)\right)$ of all zero-divisors of $M_{n}(F)$. Using the result and universal mapping property we prove that $M_{n}(F)$ is its total ring of fractions.

\section{Introduction}

Unless we state explicitly, we shall not assume that our rings are commutative, but we shall always assume that every ring has an identity. Let $R$ be a ring. An element $a \in R$ is called a zero-divisor of $R$ if there exists a non-zero element $b \in R$ such that $a b=0$. Let $Z(R)$ denote the set of all zero-divisors of $R$. Then $0 \in Z(R)$.

A commutative ring $R$ is called an integral domain if $Z(R)=\{0\}$. For example, the ring $\mathbb{Z}$ of integers is an integral domain, but $\mathbb{Z}^{2}$ is not.

\section{Localizations of Commutative Rings}

Let $A$ be a commutative ring and let $S$ be a multiplicatively closed set in $A$. Define a map $f: A \rightarrow S^{-1} A$ by $f(x)=x / 1$, where $x \in A$. Then $f$ is a ring

\footnotetext{
Received September 30, 2009. Accepted November 18, 2009.

2000 Mathematics Subject Classification: 13B30, 15A33

Key words and phrases: localizations, matrix rings, the total rings of fractions
} 
homomorphism. This is called the natural ring homomorphism.

Lemma 1.1. Let $A$ be an integral domain. Then the following are true.

(1) $A \backslash\{0\}$ is a multiplicatively closed set in $A$.

(2) $(A \backslash\{0\})^{-1} A$ is the field of fractions of $A$.

(3) If $F$ is the field of fractions of $A$, then the natural ring homomorphism $f: A \rightarrow F$ is injective.

However, the natural ring homomorphism $f: A \rightarrow S^{-1} A$ is not necessarily injective.

Let $A$ be a field and let $F$ be the field of fractions of $A$. If $s$ is a non-zero element of $A$, then

$$
s^{-1} / 1=(s / 1)^{-1}=1 / s \text { in } F
$$

Hence $A$ is isomorphic to $F$.

Let $A, B$ be commutative rings and let $f: A \rightarrow B$ be a ring homomorphism. Then $f(A)$ is a subring of $B$. However, $f(A)$ is not always an ideal of $B$.

Proposition 1.2. Let $A$ be a commutative ring and let $F$ be a field. Let $g: A \rightarrow F$ be a non-zero ring homomorphism and let $\mathfrak{p}=\operatorname{Ker}(g)$. Then the following are true.

(1) $\mathfrak{p}$ is a prime ideal of $A$.

(2) The field $A_{\mathfrak{p}} / \mathfrak{p} A_{\mathfrak{p}}$ can be embedded in the field $F$.

Lemma 1.3. Let $A$ be a commutative ring and let $I$ be an ideal of $A$. Let $S$ be a multiplicatively closed set in $A$ such that $S \cap I=\emptyset$. Then the following are true.

(1) If $\bar{S}$ is the image of $S$ under the natural homomorphism $\pi: A \rightarrow A / I$, then $\bar{S}$ is a multiplicatively closed set in $A / I$.

$(2)$

$$
\frac{S^{-1} A}{I S^{-1} A} \cong \bar{S}^{-1}(A / I) .
$$

If we use Lemma 1.3 (2) and Lemma 1.1, then we can get the following result. 
Proposition 1.4. Let $A$ be a commutative ring and let $\mathfrak{p}$ be a prime ideal of $A$. Then the following are true.

(1) $\frac{(A \backslash \mathfrak{p})+\mathfrak{p}}{\mathfrak{p}}=A / \mathfrak{p} \backslash\{0+\mathfrak{p}\}$ in $A / \mathfrak{p}$.

(2) The field of fractions of the integral domain $A / \mathfrak{p}$ is isomorphic to $A_{\mathfrak{p}} / \mathfrak{p} A_{\mathfrak{p}}$.

Proposition 1.4 (2) can be proved alternatively as follows. Let $\pi: A \rightarrow A / \mathfrak{p}$ be the natural homomorphism. Let $\varphi_{0}: A / \mathfrak{p} \rightarrow(A / \mathfrak{p})_{0}$ be the natural ring homomorphism. Then by Lemma 1.1, $\varphi_{0}: A / \mathfrak{p} \rightarrow(A / \mathfrak{p})_{0}$ is injective. Consider the composite map $g: A \stackrel{\pi}{\longrightarrow} A / \mathfrak{p} \stackrel{\varphi_{0}}{\longrightarrow}(A / \mathfrak{p})_{0}$. Then $g$ is a ring homomorphism with $\operatorname{Ker}(g)=\mathfrak{p}$. Consider the following diagram

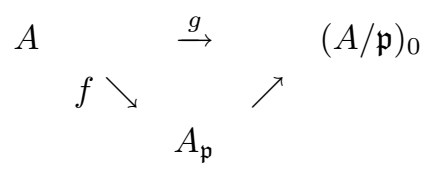

Then by the universal mapping property(see [E95, p.60]) there exists a homomorphism $h: A_{\mathfrak{p}} \rightarrow(A / \mathfrak{p})_{0}$ such that $h \circ f=g$. Further, $h$ is an epimorphism with $\operatorname{Ker}(h)=\mathfrak{p} A_{\mathfrak{p}}$. Hence, by the first isomorphism theorem for rings, $A_{\mathfrak{p}} / \mathfrak{p} A_{\mathfrak{p}} \cong(A / \mathfrak{p})_{0}$, as required.

Corollary 1.5. Let $A$ be a commutative ring. If $\mathfrak{m}$ is a maximal ideal of $A$, then $A / \mathfrak{m}$ is isomorphic to $A_{\mathfrak{m}} / \mathfrak{m} A_{\mathfrak{m}}$.

\section{Matrix Rings over Commutative Rings}

Let $R$ be a ring. An element $a \in R$ is called a unit element of $R$ if there exists an element $b \in R$ such that $a b=1, b a=1$. If $a \in R$ is a unit element of $R$, then $a \neq 0$. Let $U(R)$ denote the set of all unit elements of $R$.

If $F$ is a field, then $U(F)=F \backslash\{0\}$. For the following result, see [FIS03, Exercise 27 (a), p.231].

Proposition 2.1. Let $F$ be a field and let $A \in M_{n}(F)$. Then

$$
\operatorname{det}(\operatorname{adj} A)=(\operatorname{det} A)^{n-1} .
$$


Proof. Let $A \in M_{n}(F)$. Then $A \operatorname{adj} A=(\operatorname{det} A) I$.

Assume that $\operatorname{det} A \neq 0$. Since $\operatorname{det} A \operatorname{det}(\operatorname{adj} A)=(\operatorname{det} A)^{n}$, we have

$$
\operatorname{det}(\operatorname{adj} A)=(\operatorname{det} A)^{n-1} .
$$

Assume that $\operatorname{det} A=0$. If $A=0$, then $\operatorname{adj} A=0$ and hence

$$
\operatorname{det}(\operatorname{adj} A)=0=(\operatorname{det} A)^{n-1} .
$$

Assume that $A \neq 0$. Note that $A \operatorname{adj} A=(\operatorname{det} A) I=0$. We can prove that $\operatorname{adj} A$ is singular. For otherwise, there exit nonsingular matrices $P, Q$ such that $P(\operatorname{adj} A) Q=I$. Then

$$
A=A P^{-1} I P=A P^{-1}(P(\operatorname{adj} A) Q) P=A(\operatorname{adj} A) Q P=0 Q P=0 .
$$

This is a contradiction. Hence $\operatorname{adj} A$ is singular. Thus,

$$
\operatorname{det}(\operatorname{adj} A)=0=(\operatorname{det} A)^{n-1} .
$$

Let $R$ be a commutative ring. Let

$$
A=\left(\begin{array}{cccc}
a_{11} & a_{12} & \cdots & a_{1 n} \\
a_{21} & a_{22} & \cdots & a_{2 n} \\
\vdots & \vdots & \ddots & \vdots \\
a_{n 1} & a_{n 2} & \cdots & a_{n n}
\end{array}\right) .
$$

be in $M_{n}(R)$. For each $i, j \in\{1,2, \cdots, n\}$, let $\tilde{A}_{i, j}$ be the matrix which is obtained from $A$ by deleting the $i$-th row and $j$-th column. Then

$$
\tilde{A}_{i, j}=\left(\begin{array}{ccccccc}
a_{11} & a_{12} & \cdots & a_{1, j-1} & a_{1, j+1} & \cdots & a_{1 n} \\
a_{21} & a_{22} & \cdots & a_{2, j-1} & a_{2, j+1} & \cdots & a_{2 n} \\
\vdots & \vdots & \ddots & \vdots & \vdots & \ddots & \vdots \\
a_{i-1,1} & a_{i-1,2} & \cdots & a_{i-1, j-1} & a_{i-1, j+1} & \cdots & a_{i-1, n} \\
a_{i+1,1} & a_{i+1,2} & \cdots & a_{i+1, j-1} & a_{i+1, j+1} & \cdots & a_{i+1, n} \\
\vdots & \vdots & \ddots & \vdots & \vdots & \ddots & \vdots \\
a_{n 1} & a_{n 2} & \cdots & a_{n, j-1} & a_{n, j+1} & \cdots & a_{n n}
\end{array}\right) .
$$


For each $s \in\{1,2, \cdots, i-1, i+1, \cdots, n\}$ and $t \in\{1,2, \cdots, j-1, j+1, \cdots, n\}$, let $A_{s t}$ be the cofactor of $a_{s t}$ as an entry of the $(n-1) \times(n-1)$ matrix $\tilde{A}_{i, j}$. Then

$$
\operatorname{adj}\left(\tilde{A}_{i, j}\right)=\left(\begin{array}{ccccccc}
A_{11} & A_{21} & \cdots & A_{i-1,1} & A_{i+1,1} & \cdots & A_{n 1} \\
A_{12} & A_{22} & \cdots & A_{i-1,2} & A_{i+1,2} & \cdots & A_{n 2} \\
\vdots & \vdots & \ddots & \vdots & \vdots & \ddots & \vdots \\
A_{1, j-1} & A_{2, j-1} & \cdots & A_{i-1, j-1} & A_{i+1, j-1} & \cdots & A_{n, j-1} \\
A_{1, j+1} & A_{2, j+1} & \cdots & A_{i-1, j+1} & A_{i+1, j+1} & \cdots & A_{n, j+1} \\
\vdots & \vdots & \ddots & \vdots & \vdots & \ddots & \vdots \\
A_{1 n} & A_{2 n} & \cdots & A_{i-1, n} & A_{i+1, n} & \cdots & A_{n n}
\end{array}\right) .
$$

Now, for each $j \in\{1,2, \cdots, n\}$, construct an $n \times n$ matrix $B_{j}$ over $R$ as follows.

The entries of $j$-th row and $j$-th column of $B_{j}$ are all zero and the remaining entries of $B_{j}$ are from $\operatorname{adj}\left(\tilde{A}_{i, j}\right)$. More precisely,

$$
B_{j}=\left\{\begin{array}{ccccccc}
A_{11} & \cdots & A_{i-1,1} & 0 & A_{i+1,1} & \cdots & A_{n 1} \\
A_{12} & \cdots & A_{i-1,2} & 0 & A_{i+1,2} & \cdots & A_{n 2} \\
\vdots & \ddots & \vdots & \vdots & \vdots & \ddots & \vdots \\
A_{1, j-1} & \cdots & A_{i-1, j-1} & 0 & A_{i+1, j-1} & \cdots & A_{n, j-1} \\
0 & \cdots & 0 & 0 & 0 & \cdots & 0 \\
A_{1, j+1} & \cdots & A_{i-1, j+1} & 0 & A_{i+1, j+1} & \cdots & A_{n, j+1} \\
\vdots & \ddots & \vdots & \vdots & \vdots & \ddots & \vdots \\
A_{1 n} & \cdots & A_{i-1, n} & 0 & A_{i+1, n} & \cdots & A_{n n}
\end{array}\right) \text { if } j=i
$$

where

$$
s \begin{cases}=j & \text { if } j<i \\ \geq i+2 & \text { if } j>i .\end{cases}
$$

Then we have the following result. 
Lemma 2.2. Let $R$ be a commutative ring. Let $A \in M_{n}(R)$. For each $j \in\{1,2, \cdots, n\}$, let $B_{j}$ be constructed as above. If $\operatorname{adj}(A)=0$, then for each $j \in\{1,2, \cdots, n\}, A B_{j}=0$.

If $A \in M_{2}(R)$ and $\operatorname{adj} A=0$, then $A=0$. Now, let $A \in M_{3}(R)$. Assume that $\operatorname{adj}(A)=0$. Then for every $i, j \in\{1,2,3\}, A_{i j}=0$. Let $i, j \in\{1,2,3\}$. Then

$$
\tilde{A}_{i, j} \operatorname{adj}\left(\tilde{A}_{i, j}\right)=\operatorname{det}\left(\tilde{A}_{i j}\right) I=0
$$

since $(-1)^{i+j} \operatorname{det}\left(\tilde{A}_{i j}\right)=0$. If $\operatorname{adj}\left(\tilde{A}_{i, j}\right)=0$, then $\tilde{A}_{i, j}=0$ since $\tilde{A}_{i, j} \in M_{2}(R)$.

If for every $i, j \in\{1,2,3\} \operatorname{adj}\left(\tilde{A}_{i, j}\right)=0$, then for every $i, j \in\{1,2,3\} \tilde{A}_{i, j}=0$ and hence $A=0$. Hence, $A \in Z\left(M_{3}(R)\right)$.

If there exist $i, j \in\{1,2,3\}$ such that $\operatorname{adj}\left(\tilde{A}_{i, j}\right) \neq 0$, then $B_{j} \neq 0$. Hence by Lemma 2.2 $A \in Z\left(M_{3}(R)\right)$. Therefore if $R$ is a commutative ring, then

$$
\begin{aligned}
M_{3}(R)= & G L_{3}(R) \\
& \cup\left\{A \in M_{3}(R) \mid \operatorname{det} A \neq 0 \text { and } \operatorname{det} A \notin U(R)\right\} \\
& \cup Z\left(M_{3}(R)\right)
\end{aligned}
$$

More generally we proceed as follows.

Lemma 2.3. Let $R$ be a commutative ring. Let $A \in M_{n}(R)$. If $\operatorname{det} A=0$, then $A \in Z\left(M_{n}(R)\right)$.

Proof. Let

$$
A=\left(\begin{array}{cccc}
a_{11} & a_{12} & \cdots & a_{1 n} \\
a_{21} & a_{22} & \cdots & a_{2 n} \\
\vdots & \vdots & \ddots & \vdots \\
a_{n 1} & a_{n 2} & \cdots & a_{n n}
\end{array}\right)
$$

be in $M_{n}(R)$. For each $i, j \in\{1,2, \cdots, n\}$, let $M_{i j}$ be the minor of $A$. Denote 
elementary column operations by $\rightarrow$. Then

$$
A \rightarrow\left(\begin{array}{cccc}
a_{11} M_{11} & a_{12} & \cdots & a_{1 n} \\
a_{21} M_{11} & a_{22} & \cdots & a_{2 n} \\
\vdots & \vdots & \ddots & \vdots \\
a_{n 1} M_{11} & a_{n 2} & \cdots & a_{n n}
\end{array}\right)
$$

$$
\begin{aligned}
& \rightarrow\left(\begin{array}{cccc}
a_{11} M_{11} & a_{12} M_{12} & \cdots & a_{1 n} M_{1 n} \\
a_{21} M_{11} & a_{22} M_{12} & \cdots & a_{2 n} M_{1 n} \\
\vdots & \vdots & \ddots & \vdots \\
a_{n 1} M_{11} & a_{n 2} M_{12} & \cdots & a_{n n} M_{1 n}
\end{array}\right) \\
& \rightarrow\left(\begin{array}{cccc}
a_{11} M_{11} & \cdots & a_{1, n-1} M_{1, n-1} & a_{11} M_{11}-\cdots+(-1)^{1+n} a_{1 n} M_{1 n} \\
a_{21} M_{11} & \cdots & a_{2, n-1} M_{1, n-1} & a_{21} M_{11}-\cdots+(-1)^{2+n} a_{2 n} M_{1 n} \\
\vdots & \ddots & \vdots & \vdots \\
a_{n 1} M_{11} & \cdots & a_{n, n-1} M_{1, n-1} & a_{n 1} M_{11}-\cdots+(-1)^{n+n} a_{n n} M_{1 n}
\end{array}\right) \\
& =\left(\begin{array}{cccc}
a_{11} M_{11} & \cdots & a_{1, n-1} M_{1, n-1} & \operatorname{det} A \\
a_{21} M_{11} & \cdots & a_{2, n-1} M_{1, n-1} & 0 \\
\vdots & \ddots & \vdots & \vdots \\
a_{n 1} M_{11} & \cdots & a_{n, n-1} M_{1, n-1} & 0
\end{array}\right) \\
& =\left(\begin{array}{cccc}
a_{11} M_{11} & \cdots & a_{1, n-1} M_{1, n-1} & 0 \\
a_{21} M_{11} & \cdots & a_{2, n-1} M_{1, n-1} & 0 \\
\vdots & \ddots & \vdots & \vdots \\
a_{n 1} M_{11} & \cdots & a_{n, n-1} M_{1, n-1} & 0
\end{array}\right) .
\end{aligned}
$$

So, there exists a non-singular matrix $P \in M_{n}(R)$ such that

$$
A P=\left(\begin{array}{cccc}
a_{11} M_{11} & \cdots & a_{1, n-1} M_{1, n-1} & 0 \\
a_{21} M_{11} & \cdots & a_{2, n-1} M_{1, n-1} & 0 \\
\vdots & \vdots & \ddots & \vdots \\
a_{n 1} M_{11} & \cdots & a_{n, n-1} M_{1, n-1} & 0
\end{array}\right)
$$


Let $E_{i j}$ be the matrix in which the only non-zero entry is a 1 in the $i$ th row and $j$ th column. Then

$$
\begin{aligned}
A P= & M_{11}\left(a_{11} E_{11}+a_{21} E_{21}+\cdots+a_{n 1} E_{n 1}\right) \\
& +\cdots \\
& +M_{1, n-1}\left(a_{1, n-1} E_{1, n-1}+a_{2, n-1} E_{2, n-1}+\cdots+a_{n, n-1} E_{n, n-1}\right) .
\end{aligned}
$$

Multiplying both sides of this equation by $E_{n n}$, we have $A P E_{n n}=0$. Since $P E_{n n} \neq 0$, it follows that $A \in Z\left(M_{n}(R)\right)$.

If $R$ is a commutative ring, then $U\left(M_{n}(R)\right)$ forms a group under the matrix multiplication. This group is called the general linear group of degree $n$ over $R$ and is denoted by $G L_{n}(R)$. Since $A \in U\left(M_{n}(R)\right)$ if and only if $\operatorname{det} A \in U(R)$, we have

$$
G L_{n}(R)=\left\{A \in M_{n}(R) \mid \operatorname{det} A \in U(R)\right\} .
$$

ThEOREM 2.4. Let $R$ be a commutative ring. Then

$$
\begin{aligned}
M_{n}(R)= & G L_{n}(R) \\
& \cup\left\{A \in M_{n}(R) \mid \operatorname{det} A \neq 0 \text { and } \operatorname{det} A \notin U(R)\right\} \\
& \cup Z\left(M_{n}(R)\right)
\end{aligned}
$$

Proof. Let $A \in M_{n}(R)$. Then either $\operatorname{det} A \in U(R)$ or $\operatorname{det} A \notin U(R)$. If $\operatorname{det} A \in U(R)$, then $A \in G L_{n}(R)$. Assume that $\operatorname{det} A \notin U(R)$. If $\operatorname{det} A \neq 0$, then $A \in\left\{A \in M_{n}(R) \mid \operatorname{det} A \neq 0\right.$ and $\left.\operatorname{det} A \notin U(R)\right\}$. If $\operatorname{det} A=0$, then by Lemma $2.3 A \in Z\left(M_{n}(R)\right)$.

\section{The Total Rings of Fractions of Matrix Rings}

LEMma 3.1. If $R$ is a ring, then

$$
U(R) \cap Z(R)=\emptyset .
$$


Proof. Suppose that $U(R) \cap Z(R) \neq \emptyset$. Take an element $a \in U(R) \cap Z(R)$. Then $a \in U(R)$, so there exists an element $b \in R$ such that $a b=1, b a=1$. $a \in Z(R)$, so there exists a non-zero element $c \in R$ such that $a c=0$. Then

$$
c=1 c=(b a) c=b(a c)=b 0=0 .
$$

This contradiction shows that $U(R) \cap Z(R)=\emptyset$.

Definition. Let $R$ be a ring (not necessarily commutative). A multiplicatively closed subset $S$ of $R$ is said to be saturated if whenever $x y \in S$, where $x$, $y \in R$, then $x \in S$ and $y \in S$.

Note that this definition in non-commutative case coincides completely with that in commutative case.

Theorem 3.2. Let $R$ be a unique factorization domain and let

$$
S=U(R) \cup\{a \in R \mid a \text { is a product of principal primes of } R\} .
$$

Then

(1) $S$ is a saturated multiplicatively closed set in $R$.

(2) $M_{n}(R)=\left\{A \in M_{n}(R) \mid \operatorname{det} A \in S\right\} \cup Z\left(M_{n}(R)\right)$.

(3) $M_{n}(R)_{S}=U\left(M_{n}(R)_{S}\right) \dot{\cup} Z\left(M_{n}(R)_{S}\right)$.

(4) $U\left(M_{n}(R)_{S}\right)$ is a subgroup of $G L_{n}\left(R_{S}\right)$.

(5) $M_{n}(R)_{S}$ is $R_{S}$-isomorphic to $M_{n}\left(R_{S}\right)$.

Proof. (1) See [K74]. (2) By Theorem 2.4. (3) Let $A / s$ be any element of $M_{n}(R)_{S}$. Assume $\operatorname{det} A \in S$. Then

$$
\begin{aligned}
& (A / s)(s \operatorname{adj} A / \operatorname{det} A))=I / 1, \\
& (s \operatorname{adj} A / \operatorname{det} A))(A / s)=I / 1 .
\end{aligned}
$$

So, $\left.(A / s)^{-1}=s \operatorname{adj} A / \operatorname{det} A\right) \in M_{n}(R)_{S}$. Hence $A / s \in U\left(M_{n}(R)_{S}\right)$. Or, assume $\operatorname{det} A \notin S$. Then by (2) $A \in Z\left(M_{n}(A)\right)$. Hence $A / s \in Z\left(M_{n}(A)_{S}\right)$. Therefore it follows from Lemma 3.1 that (3) holds. 
(4) Define a map $\varphi: M_{n}(R)_{S} \rightarrow M_{n}\left(R_{S}\right)$ by

$$
\varphi\left(\left(\begin{array}{cccc}
a_{11} & a_{12} & \cdots & a_{1 n} \\
a_{21} & a_{22} & \cdots & a_{2 n} \\
\vdots & \vdots & \ddots & \vdots \\
a_{n 1} & a_{n 2} & \cdots & a_{n n}
\end{array}\right) / s\right)=\left(\begin{array}{cccc}
a_{11} / s & a_{12} / s & \cdots & a_{1 n} / s \\
a_{21} / s & a_{22} / s & \cdots & a_{2 n} / s \\
\vdots & \vdots & \ddots & \vdots \\
a_{n 1} / s & a_{n 2} / s & \cdots & a_{n n} / s
\end{array}\right)
$$

Then $\varphi$ is a well-defined injective ring homomorphism. Hence, $U\left(M_{n}(R)_{S}\right)$ is a subgroup of $G L_{n}\left(R_{S}\right)$.

(5)

$$
\begin{aligned}
M_{n}(R)_{S} & \cong M_{n}(R) \otimes_{R} R_{S} \\
& \cong R^{n^{2}} \otimes_{R} R_{S} \\
& \cong(R \oplus R \oplus \cdots \oplus R) \otimes_{R} R_{S} \\
& \cong\left(R \otimes_{R} R_{S}\right) \oplus\left(R \otimes_{R} R_{S}\right) \oplus \cdots \oplus\left(R \otimes_{R} R_{S}\right) \\
& \cong\left(R \otimes_{R} R_{S}\right)^{n^{2}} \\
& \cong R_{S}^{n^{2}} \\
& \cong M_{n}\left(R_{S}\right) .
\end{aligned}
$$

The following result comes from Theorem 2.4 and Lemma 3.1. However, we give an alternative proof.

Theorem 3.3. Let $F$ be a field. Then $M_{n}(F)=G L_{n}(F) \dot{\cup} Z\left(M_{n}(F)\right)$.

Proof. It is clear that the result holds for $n=1$.

Let $n \geq 2$. Assume $A=0$. Then $A I=A=0$. Hence $A \in Z\left(M_{n}(F)\right)$.

Assume $A \neq 0$. Let $E_{i j}$ be the matrix in which the only non-zero entry is a 1 in the ith row and jth column. Then by [K96, Theorem 1.20, p.65], $A$ is equivalent to $E_{11}+E_{22}+\cdots+E_{r r}$ for some $r \in\{1,2,3, \cdots, n\}$. There exist nonsingular matrices $P, Q$ such that

$$
P A Q=E_{11}+E_{22}+\cdots+E_{r r}
$$

If $r=n$, then $P A Q=E_{11}+E_{22}+\cdots+E_{n n}=I$ and hence $\operatorname{det} A \neq 0$. Thus, $A \in G L_{n}(F)$. Assume $r<n$. Multiplying $E_{r+1,1}$ on both sides of the equation 
$(*)$, we have

$$
\begin{aligned}
P A Q E_{r+1,1} & =E_{11} E_{r+1,1}+E_{22} E_{r+1,1}+\cdots+E_{r r} E_{r+1,1} \\
& =\delta_{1, r+1} E_{11}+\delta_{2, r+1} E_{21}+\cdots+\delta_{r, r+1} E_{r 1} \\
& =0
\end{aligned}
$$

Hence $A Q E_{r+1,1}=0$ and $Q E_{r+1,1} \neq 0$. Thus $A \in Z\left(M_{n}(F)\right)$. Therefore $M_{n}(F)=G L_{n}(F) \cup Z\left(M_{n}(F)\right)$. It follows from Lemma 3.1 that

$$
M_{n}(F)=G L_{n}(F) \dot{\cup} Z\left(M_{n}(F)\right) .
$$

THEOREM 3.4. If $F$ is a field, then the matrix ring $M_{n}(F)$ is its total ring of fractions.

Proof. Assume that $F$ is a field. Then

$$
G L_{n}(F)=\left\{A \in M_{n}(F) \mid \operatorname{det}(A) \neq 0\right\}=U\left(M_{n}(F)\right) .
$$

Hence it is clear that $G_{n}(F)$ is a saturated multiplicatively closed subset of $M_{n}(F)$. Let $S_{0}=M_{n}(F) \backslash Z\left(M_{n}(F)\right)$. Then by Theorem 3.3, $S_{0}=G L_{n}(F)$ and hence $S_{0}$ is a saturated multiplicatively closed subset of $M_{n}(F)$. Thus $M_{n}(F)_{S_{0}}$ is the total ring of fractions of $M_{n}(F)$ ([AM69, Chapter 3, Exercise 9, p.44] and [H88].) Let $i d: M_{n}(F) \rightarrow M_{n}(F)$ be the identity ring homomorphism. Since

$$
S_{0}=G L_{n}(F)=U\left(M_{n}(F)\right),
$$

we can see that $i d(A)$ is a unit in $M_{n}(F)$ for all $A \in S_{0}$. Consider the following diagram

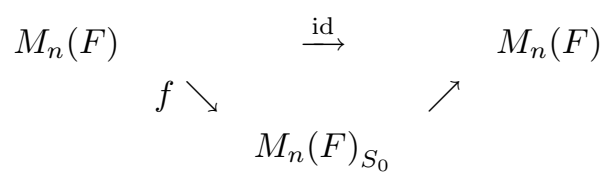

Then by the universal mapping property (see [E95, p.60]) there exists a homomorphism $h: M_{n}(F)_{S_{0}} \rightarrow M_{n}(F)$ such that $h \circ f=$ id. From this equation, it follows that $h$ is an epimorphism. Further, for any $A / B \in M_{n}(F)_{S_{0}}$,

$$
h(A / B)=h(A / I \cdot I / B)=h(f(A)) h\left(f\left(B^{-1}\right)\right)=i d(A) i d\left(B^{-1}\right)=A B^{-1} .
$$


From this equation we can see that $h$ is injective. Hence $h$ is bijective. Thus $h$ is an isomorphism. This shows that $M_{n}(F) \cong M_{n}(F)_{S_{0}}$. Therefore $M_{n}(F)$ is its total ring of fractions.

Let $\mathbb{C}$ be the complex number field. Then it is clear that $M_{n}(\mathbb{C})$ is isomorphic to $M_{n}(\mathbb{C})_{G L_{n}(\mathbb{C})}$ because $G L_{n}(\mathbb{C})=U\left(M_{n}(\mathbb{C})\right.$ ). If we let $S_{0}=$ $M_{n}(\mathbb{C}) \backslash Z\left(M_{n}(\mathbb{C})\right)$, then it follows from Theorem 3.3 that

$$
M_{n}(\mathbb{C}) \cong M_{n}(\mathbb{C})_{S_{0}} .
$$

Hence $M_{n}(\mathbb{C})$ is its total ring of fractions. In fact, the proof of Theorem 3.4 is standard.

\section{Acknowledgements}

The author would like to thank Professor Richard Green and the referees for their comments on the author's draft. They strongly suggested rewriting chapter 1 before the author submits to a journal.

\section{References}

[AM69] M. F. Atiyah and I. G. MacDonald, Introduction to Commutative Algebra, Addison-Wesley, Reading, Mass., 1969.

[E95] D. Eisenbud, Commutative Algebra with a View toward Algebraic Geometry, Springer-Verlag New York, Inc., 1995.

[FIS03] S. H. Friedberg, A. J. Insel and L. E. Spence, Linear Algebra, Fourth Edition, Pearson Education, Inc., 2003.

[K96] B. Kolman, Elementary Linear Algebra, Sixth Edition, Prentice-Hall, Inc., 1996. 
[K74] I. Kaplansky, Commutative Rings, The University of Chicago Press, Revised Edition, 1974.

[H88] J. A. Huckaba, Commutative Rings with Zero Divisors, Marcel Dekker, Inc., 1988.

Sang Cheol Lee

Department of Mathematics Education

Chonbuk National University

Chonju, Chonbuk 561-756, Korea

E-mail: scl@chonbuk.ac.kr

and

Department of Mathematics

The University of Colorado at Boulder

395 UCB

Boulder, Colorado 80309-0395, USA

E-mail: Sang.C.Lee@Colorado.EDU 